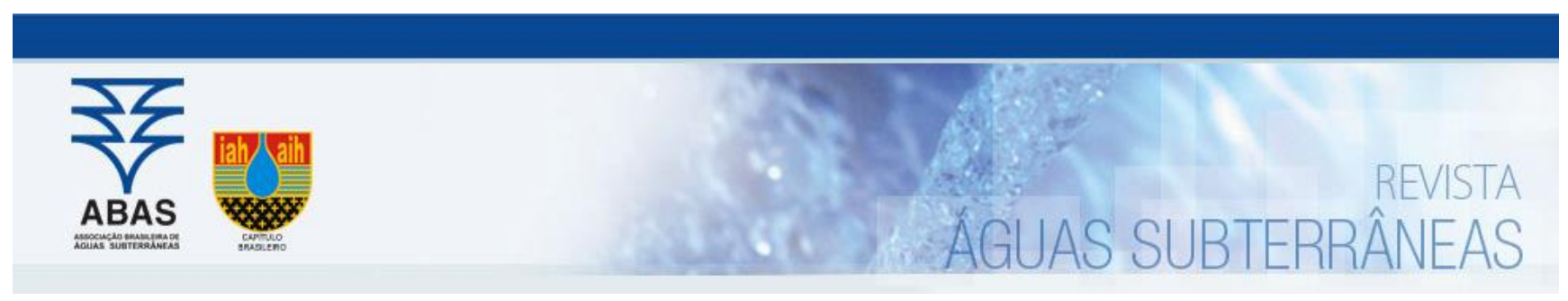

Artigos

\title{
Simulações da migração de plumas dissolvidas de compostos BTEX geradas por LNAPL trapeado
}

\section{Simulations of dissolved BTEX plumes migration derived from entrapped LNAPL}

\section{Elias Hideo Teramoto1; Marcus Paulus Martins Baessa2; Hung Kiang Chang ${ }^{3}$}

1 Laboratório de Estudos de Bacias (Lebac) -UNESP- Campus de Rio Claro, SP / Centro de Estudos Ambientais (CEA), UNESP UNESP- Campus de Rio Claro, SP.

2 Centro de Pesquisas Leopoldo Américo Miguez de Mello (CENPES), Petrobras.

3 Departamento de Geologia Aplicada - UNESP- Campus de Rio Claro, SP / Laboratório de Estudos de Bacias (Lebac) -UNESPCampus de Rio Claro, SP / Centro de Estudos Ambientais (CEA), UNESP UNESP- Campus de Rio Claro, SP

凶 elias.hideo-teramoto@unesp.br, marcus.baessa@petrobras.com.br, chang@rc.unesp.br

\section{Palavras-chave:}

Depleção do LNAPL.

Plumas dissolvidas de compostos BTEX. Simulação numérica de transporte de solutos.

\section{Keywords:}

LNAPL depletion.

Dissolved plumes of BTEX compounds. Numerical simulation of contaminant transport.

\section{Revisado por pares.}

Recebido em: 05/04/2019.

Aprovado em: 10/06/2019.
Resumo

O vazamento acidental de hidrocarbonetos é um cenário comum de contaminação subsuperficial. Uma vez liberado, o hidrocarboneto migra até atingir as vizinhanças da zona saturada, onde se acumula. Em razão da flutuação do nível d'água, o LNAPL (light non-aqueous phase) é redistribuído e trapeado na zona saturada. O LNAPL trapeado é responsável pela liberação de benzeno, tolueno, etilbenzeno e xilenos (BTEX) para a água, gerando plumas de fase dissolvida desses compostos. Essas plumas variam em dimensão e concentração ao longo do tempo, em resposta à depleção dos compostos BTEX na área-fonte. Para representar esta condição é proposta uma metodologia que combina o uso dos aplicativos MODFLOW, para simulações de fluxo, EXHAURIS, para simulação da transferência de massa e MT3DMS, para simulação do transporte de solutos. As simulações realizadas reproduziram o comportamento das plumas dissolvidas de benzeno e etilbenzeno geradas a partir da dissolução do querosene de aviação. Os resultados indicam que a pluma de benzeno se extingue dentro de um período de 10 anos, enquanto o etilbenzeno persiste por mais de 20 anos. Tal diferença está associada à menor fração molar e à maior solubilidade do benzeno em relação ao etilbenzeno. Os testes indicam que a metodologia proposta é uma alternativa promissora para previsões do comportamento de plumas dissolvidas ao longo do tempo, contemplando a condição de depleção da áreafonte.

\section{Abstract}

Accidental leakage of hydrocarbons represents a common scenario of subsurfacial contamination. Once released, the hydrocarbon migrates until it reaches the vicinity of the uppermost portion of saturated zone, where it accumulates. Due to the fluctuation of the water level, the LNAPL (light non-aqueous phase) is redistributed and entrapped in the saturated zone. The entrapped LNAPL is responsible for the release of benzene, toluene, ethylbenzene and xylenes (BTEX) into the water generating the dissolved phase plumes of these compounds. The generated BTEX plumes vary in size and concentration over time in response to the depletion of BTEX compounds in the source zone. To represent this condition, a methodology was proposed that combines the use of the MODFLOW application for flow simulations, EXHAURIS to simulate mass transfer and MT3DMS to simulate solutes transport. The performed simulations were capable to reproduce the behavior of dissolved benzene and ethylbenzene plumes generated by jet fuel dissolution. Our results indicate that the benzene plume disappears within a period of 10 years, while the lifespan of ethylbenzene plume exceeds 20 years. This difference is related to the lower molar fraction and greater solubility of benzene as compared to ethylbenzene. The tests indicate that the proposed methodology is a promising alternative to predict the behavior of dissolved plumes over time contemplating the condition of depletion of the source zone.

DOI: http:/dx.doi.org/10.14295/ras.v33i3.29529

\section{INTRODUÇÃO}

O vazamento acidental de combustíveis derivados de petróleo a partir de tanques de armazenamento ou dutos de transferência representa uma das principais fontes de contaminação subsuperficial. O combustível migra na zona não-saturada até alcançar as vizinhanças da zona saturada, onde se acumula formando o LNAPL (Light Non-Aqueous Phase Liquid). A flutu- ação do nível da água (NA), em resposta à alternância cíclica de estações secas e chuvosas, induz a redistribuição vertical do LNAPL à medida que migra para partes inferiores do aquífero, quando o nível da água rebaixa (KEMBLOWSKI \& CHIANG, 1990, MARINELLI \& DURNFORD, 1996; JEONG \& CHARBENEAU, 2014). Quando o NA sobe, o LNAPL é retido pela força capilar (além de forças viscosas e de gravidade) na zona saturada, gerando um fenômeno conhecido como trapea- 
mento (FARR et al., 1990, MARINELLI \& DURNFORD, 1996, KEMBLOWSKI \& CHIANG, 1990, JEONG \& CHARBENEAU, 2014; LENHARD et al., 2018). A condição de trapeamento foi demonstrada por incontáveis trabalhos, incluindo Pede (2009), Suthersan et al. (2015), Flores et al. (2016), Gatsios et al. (2018) e Isler et al. (2018). Quando ocorre o trapeamento, o LNAPL é aprisionado por capilaridade na zona saturada; em contraste, quando o NA desce, o LNAPL é liberado, e as gotas e gânglios anteriormente isolados se aglutinam e ganham mobilidade.

Uma vez trapeado, o LNAPL passa a liberar compostos solúveis para a água subterrânea, em particular benzeno, etilbenzeno, tolueno e xilenos (BTEX), que representam os compostos mais solúveis. A liberação dos compostos solúveis do LNAPL para a água se dá a partir de transferência de massa por taxa limitada (condição de não-equilíbrio), que representa um fenômeno difusivo. A massa total que é transferida do LNAPL para a água subterrânea pode ser calculada multiplicando-se o gradiente de concentração entre a água e a solubilidade efetiva do composto pelo coeficiente de transferência de massa e a área de interface específica entre as fases (MILLER et al., 1990):

$$
\mathrm{J}=\frac{d C_{a}}{d t}=k_{a} a_{n w}\left(C_{s}-C\right)
$$

Onde $\mathrm{J}$ = fluxo de massa do LNAPL residual para a água (ML 2T-1), $k_{a}=$ coeficiente de transferência de massa $\left(\mathrm{LT}^{-1}\right), C_{s}=$ máxima concentração do composto solúvel na fase aquosa (ML-3), $C$ = concentração do composto solúvel na fase aquosa $\left(\mathrm{ML}^{-3}\right)$ e $a_{n w}=$ área interfacial específica entre a água e o LNAPL (L2).

A transferência de massa ocorre até que o equilíbrio de concentração entre a água e a fase líquida não-aquosa (NAPL) seja atingido. O NAPL representa uma mistura complexa de compostos orgânicos com diferentes valores de solubilidade, o que torna a sua dissolução mais complexa que a de um composto simples. A concentração de equilíbrio depende da composição da mistura, como demonstrado por Banerjee (1984) e Mackay et al. (1991). A solubilidade de cada composto é menor que a solubilidade das substâncias puras em água e pode ser determinada em um meio poroso com dois líquidos imisciveis usando a lei de Raoult:

$$
C_{i, s a t}=\chi_{i, o} \gamma_{i, o} S_{i}
$$

Onde $C_{i, s a t}=$ concentração do composto i na água ( $\mathrm{mol} / \mathrm{L}$ ), $\chi_{i, o}=$ fração molar do composto $\mathrm{i}$ na mistura, $\gamma_{i, o}=$ coeficiente de atividade do composto $i$ na mistura e $S_{i}=$ solubilidade máxima do composto $i$ na água ( $\mathrm{mol} / \mathrm{L}$ ).

Em razão de variações nos processos que controlam a transferência de massa interfases dentro da área-fonte, ocorrem fortes variações da concentração na área-fonte ao longo do tempo, com tendência de queda. Tal tendência decorre de vários fatores, tais como saturação do LNAPL, velocidade de fluxo e taxas de biodegradação na fase aquosa. Thornton et al. (2013), Kulkarni et al. (2015), Garg et al. (2017), Teramoto \& Chang (2017), Mackay et al. (2018), dentre outros, demonstraram que, em razão da contínua perda dos compostos solúveis para a água, ocorre uma contínua depleção do LNAPL na área-fonte. Como demonstrado por Connor et al. (2015), em razão das variações de concentração na área-fonte, as plumas de fase dissolvida apresentam quatro estágios de evolução (Figura 1):

a) Expansão - fase na qual a pluma de fase dissolvida experimenta um período de crescimento dos seus limites ou de suas concentrações por movimento advectivo do soluto;

b) Estabilidade - fase na qual a pluma dissolvida atinge suas dimensões máximas, limitadas pelas taxas de biodegradação;

c) Encolhimento - fase na qual ocorre uma queda das concentrações e retração dos limites da pluma;

d) Exaurimento - fase na qual a pluma de fase dissolvida atinge concentrações abaixo do limite de detecção, em razão da completa depleção da área-fonte.

Figura 1 - Ilustração esquemática da evolução da pluma de fase dissolvida após o cessamento do vazamento

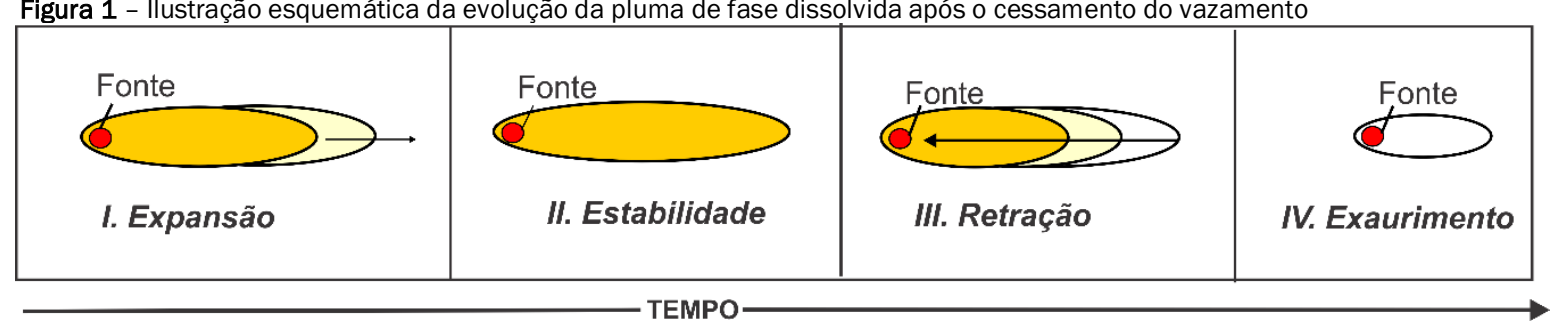

Fonte: Modificado de Connor et al. (2015)

Para a simulação de cenários mais realistas de transporte de solutos é necessário contemplar o efeito da redução da pluma dissolvida em função da depleção de compostos monoaromáticos na área-fonte. Nesse contexto, este trabalho apresenta uma metodologia que permite a simulação do transporte de compostos monoaromáticos, utilizando modelos capazes de prever a depleção da área-fonte.

\section{MATERIAIS E MÉTODOS}

\subsection{Modelo de depleção do LNAPL}

A previsão da depleção do LNAPL foi empregado o aplicativo EXHAURIS, desenvolvido a partir do modelo numérico proposto por Teramoto \& Chang (2017). O modelo proposto por esses autores, descrito pelas Eq. 3 a 6, foi desenvolvido para 
simular a depleção do LNAPL a partir da modificação do modelo proposto por Liu et al. (2012) para simular a transferência de massa dentro de um volume elementar representativo.

$$
C_{i, e q, t}=\gamma_{i}\left(M_{i, t} / \sum_{j=1}^{m} M_{j, t}\right) \cdot S_{i, s u b}
$$

$E_{i, t}=A \cdot k_{i} \cdot\left(C_{i, e q, t}-C_{i, t}\right)$

$M_{i, t}=M_{i, t-1}-\frac{\Delta t}{2} \cdot\left(E_{i, t}+E_{i, t-1}\right)$

$$
\begin{aligned}
& C_{i, t}= \\
& \frac{\left(C_{i, t-1} e^{-\lambda \frac{\Delta t}{2}}\right)+\frac{\Delta t}{2 V_{w}}\left(A \cdot k_{i} \cdot C_{i, e q, t-1}+E_{i, t-1}-Q \cdot C_{i, t-1} e^{-\lambda \frac{\Delta t}{2}}\right)}{1+\frac{\Delta t}{2 V_{w}} \cdot\left(Q+A \cdot k_{i}\right)}
\end{aligned}
$$

Onde $C_{i, t}=$ concentração do composto i na fase aquosa, $C_{i, e q, t}$ = concentração de equilíbrio do composto i, $M_{i, t}=$ massa do composto i (M), $\gamma_{i}=$ coeficiente de atividade do composto i, $V_{w}=$ volume de água no volume elementar representativo, $k_{i}$ = coeficiente de transferência de massa $\left(\mathrm{L} \mathrm{T}^{-1}\right), Q=$ fluxo ( $\mathrm{L}^{3} \mathrm{~T}$ 1), $A$ = área interfacial ( $\left.\mathrm{L}^{2}\right), S_{i, s u b}=$ solubilidade do composto i (M/L3), $E_{i, t}=$ termo de troca do composto i $(\mathrm{M} / \mathrm{T})$ e $\lambda=$ coeficiente de decaimento de primeira ordem. Os valores de $C_{i}, C_{i, e q}$, $M_{i}$, e $E_{i}$, são marcados pelo subscrito t e t-1 para indicar o passo de tempo atual e o anterior, respectivamente.
O sistema não-linear de equações 3 a 6 é solucionado numericamente empregando o método iterativo de Gauss-Seidel, com ponderação central do tempo até que a convergência seja alcançada. O modelo de Teramoto \& Chang (2017) foi desenvolvido para reproduzir a transferência de massa dentro de um volume elementar representativo (VER) do aquífero, contemplando o efeito da flutuação do nível d'água. De acordo com a formulação de Teramoto \& Chang (2017), a flutuação do NA pode promover o incremento ou decremento das concentrações na fase aquosa, dependendo da distribuição do LNAPL.

Utilizando-se o aplicativo EXHAURIS foi calculada a queda nas concentrações dos compostos monoaromáticos na fase aquosa dentro da área-fonte, tomando-se por base a queda das concentrações desses compostos no LNAPL e, consequentemente, na queda da fração molar e solubilidade efetiva desses compostos.

\subsection{Estratégia de acoplamento do modelo de depleção com modelos de transporte}

Para simular o transporte de compostos BTEX associado a uma fonte depletiva, é proposta uma estratégia metodológica, ilustrada na Figura 2, combinando o uso dos aplicativos MODFLOW 2005 (HARBAUGH, 2005), MT3DMS (ZHENG \& WANG, 1999) e EXHAURIS.

Figura 2 -Esquema ilustrando a integração do EXHAURIS, MODFLOW e MT3DMS para simulação de transporte de solutos a partir de uma fonte depletiva.

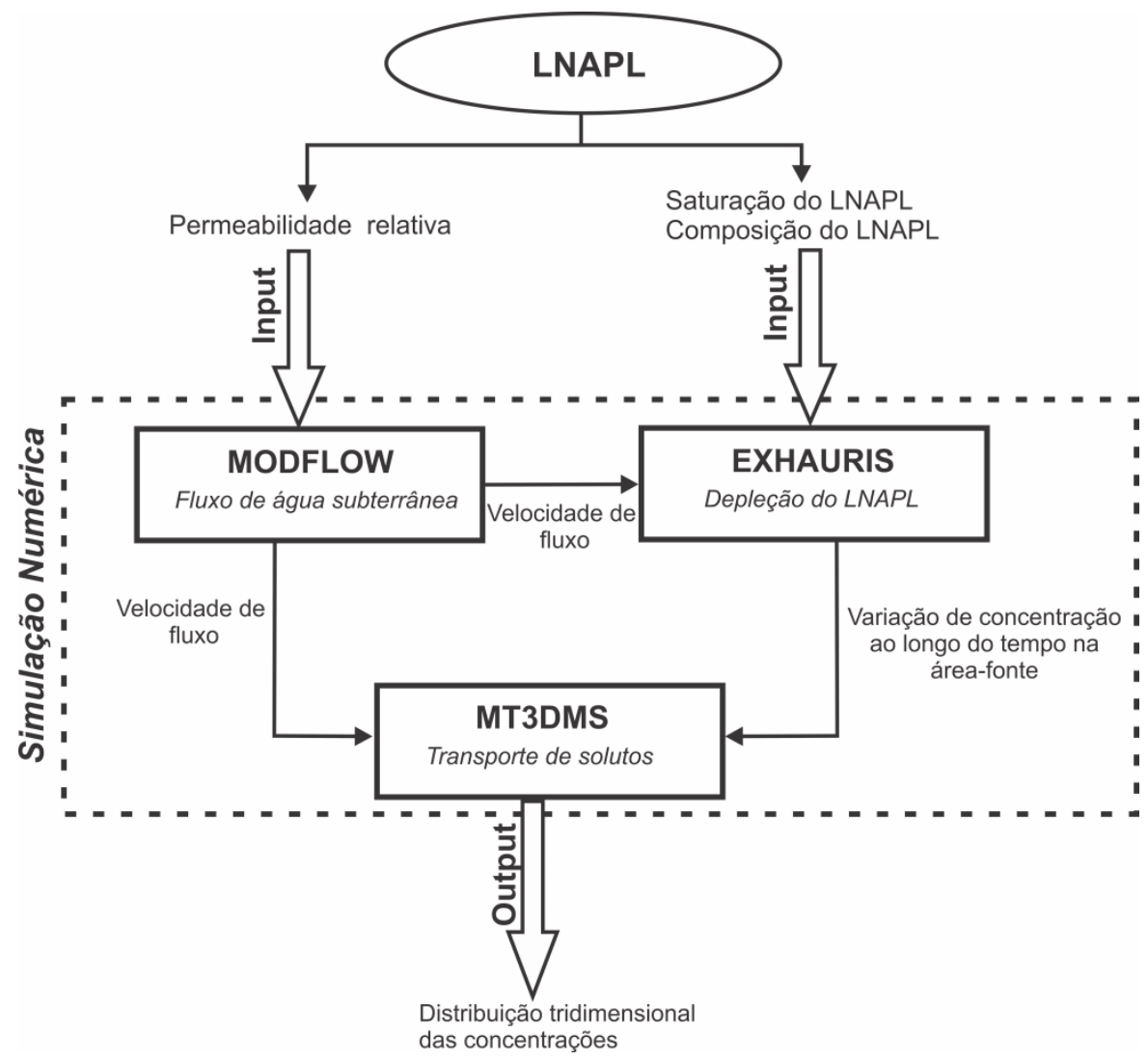


O MODFLOW 2005 emprega o método de Diferenças Finitas para simular o fluxo no meio poroso. A partir das simulações de fluxo em regime transiente são gerados os valores de taxas de fluxo para cada célula da área-fonte. Com base nas taxas de fluxo, nas propriedades do meio poroso e na quantidade de LNAPL aí presente, é calculada a redução da fração molar dos compostos monoaromáticos no LNAPL e, consequentemente, as reduções na solubilidade efetiva e na concentração desses compostos na fase aquosa, por meio do EXHAURIS. Nas simulações realizadas no EXHAURIS foi assumido a premissa que a área-interfacial, bem como a massa e o volume de LNAPL permaneciam constantes, uma vez que a fração molar destes compostos no querosene de aviação é bastante reduzida.

As concentrações assim obtidas são fornecidas ao aplicativo MT3DMS, que simula o transporte de soluto empregando o Método de Diferenças Finitas, com condições de contorno de concentração especificadas. A partir das velocidades de fluxo calculadas pelo MODFLOW e das concentrações na área-fonte fornecidas pelo EXHAURIS, são realizadas simulações de transporte de solutos no período de interesse.

\subsection{Aplicação em um modelo sintético}

Para demonstrar a aplicação da metodologia proposta na ava- liação do comportamento da fase dissolvida em razão da depleção do LNAPL, foram realizadas simulações em um modelo sintético. Este modelo foi parametrizado com informações provenientes de diversos estudos conduzidos em uma área do contaminada por querosene de aviação situada no munícipio de Paulínia (SP); esta área foi descrita por Pede (2009), Teramoto (2015), Teramoto \& Chang (2017), Teramoto et al. (2017), Teramoto \& Chang (2018), Isler et al. (2018) e Teramoto \& Chang (2019).

Com o conjunto de informações obtidas dos referidos trabaIho, foi parametrizado o modelo sintético construído, com valores de condutividade hidráulica, porosidade efetiva, gradiente hidráulico, distribuição e saturação do LNAPL e fração molar dos compostos BTEX no LNAPL.

Como exposto por Teramoto \& Chang (2017), observa-se uma tendência das elevadas concentrações serem observadas no centro da área-fonte, declinando progressivamente em direção às bordas. Com base nos resultados dos ensaios de curva de retenção apresentados por Pede (2009) e dos valores máximos de espessura de fase livre medidos nos poços de monitoramento, foram calculados os perfis de saturação de LNAPL no centro e na borda da área-fonte, onde as saturações do LNAPL são respectivamente elevadas e reduzidas.

Figura 4 - Perfil de saturação do LNAPL calculado a partir do modelo de Jeong \& Chaberneau (2014): a) Elevadas

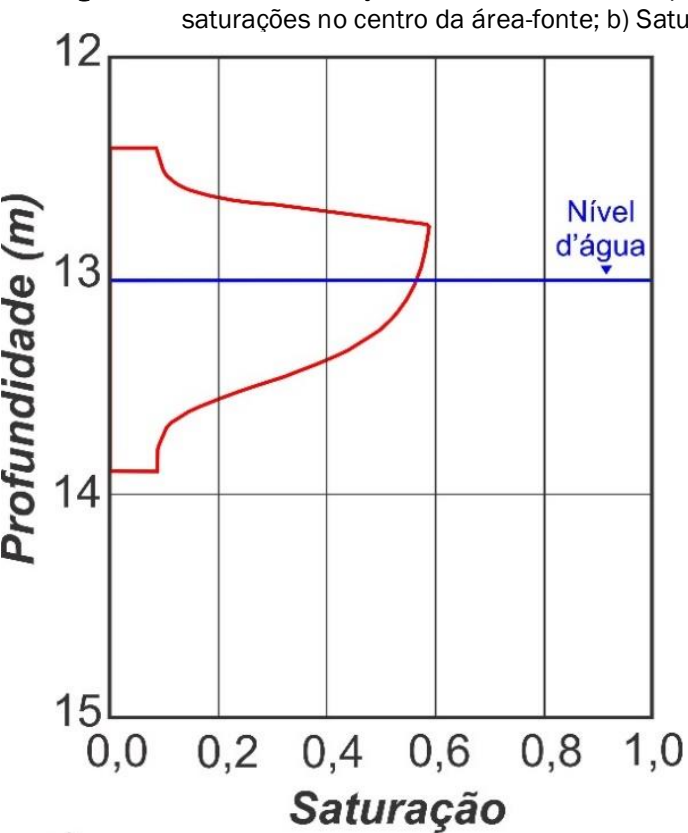

a)

As investigações com o emprego de LIF (Laser Induced Fluorescence), conduzidas por Isler et al. (2018), verificaram que o LNAPL na área considerada está trapeado no intervalo de 1 m a 2 m abaixo do nível d'água, dependendo do período avaliado. A condição de trapeamento implica em um modelo conceitual em que o LNAPL libera BTEX para a água unicamente

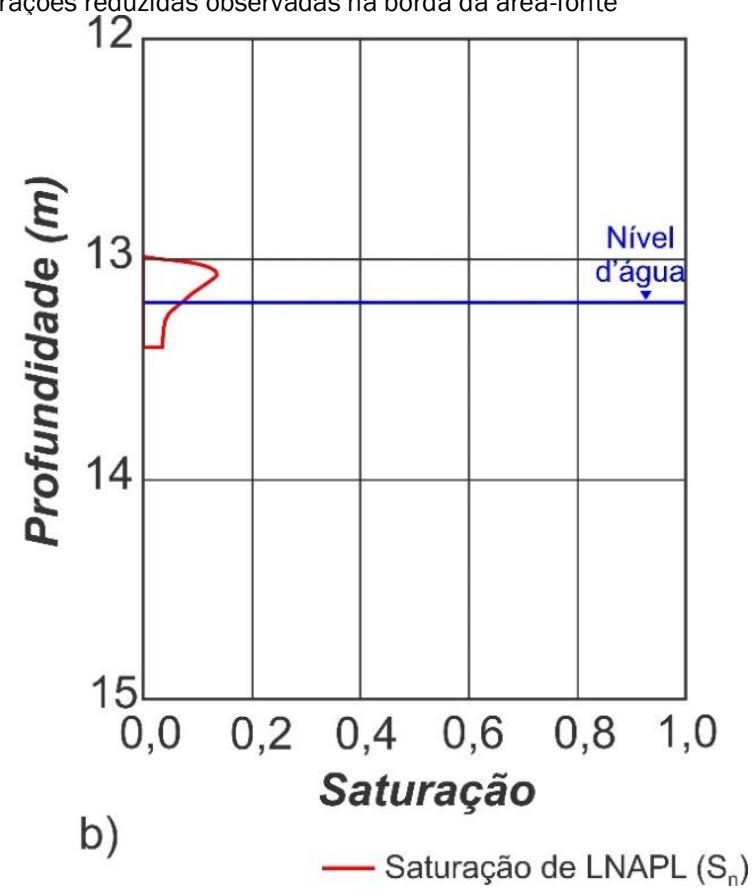

por difusão. Visando manter a simplicidade do modelo simulado, foi assumido um domínio retangular com $400 \mathrm{~m}$ de comprimento, $300 \mathrm{~m}$ de largura e $2 \mathrm{~m}$ de espessura, discretizado em 400 linhas por 500 colunas e 3 camadas (Figura 3). 
Figura 3 - Domínio do modelo simulado: a) Representação tridimensional; b) Visão em planta com os limites da área-fonte e distribuição de saturação do LNAPL na área-fonte

a)
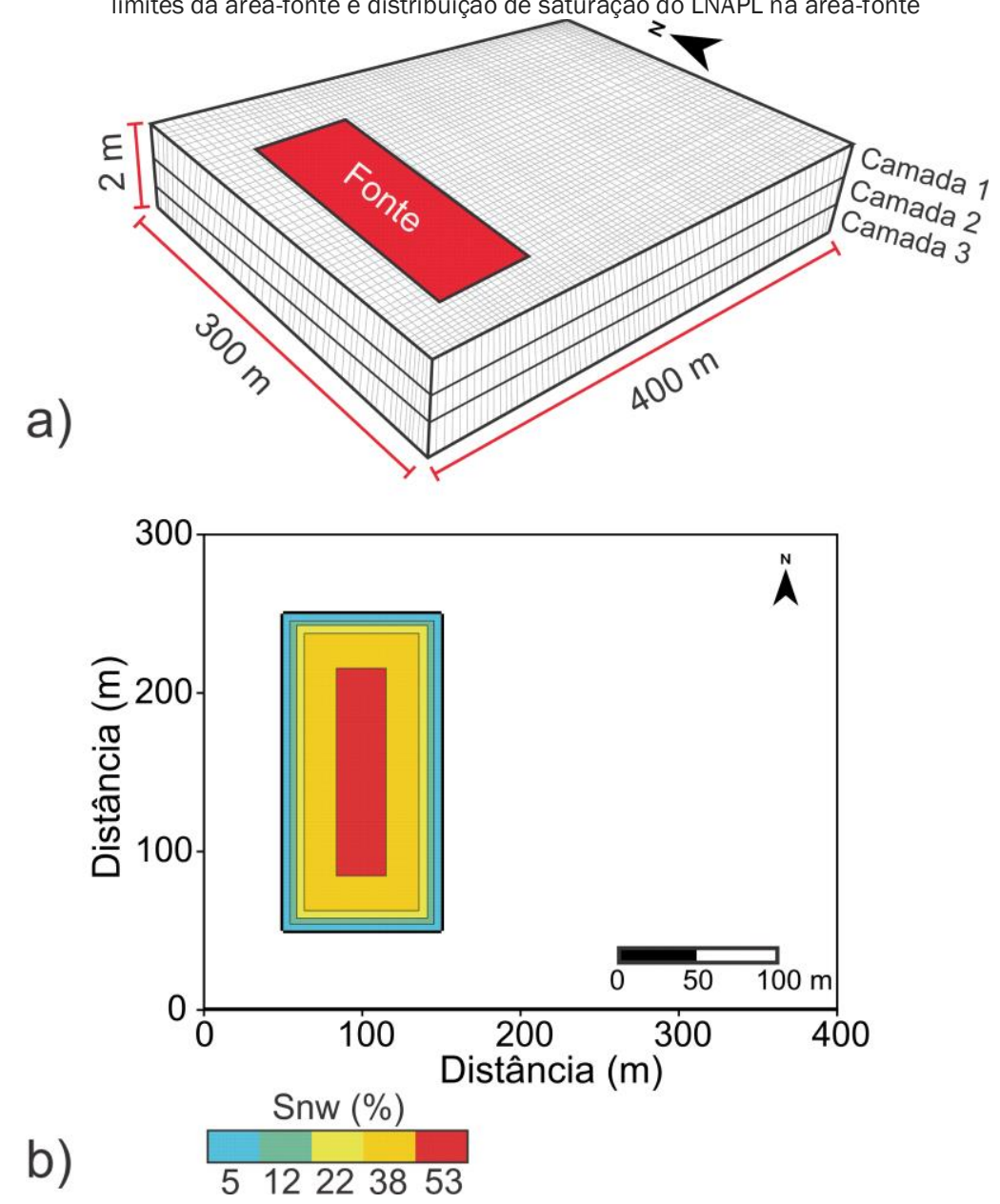

Dado que, como verificado por Teramoto \& Chang (2017), as saturações mais elevadas de LNAPL são observadas no centro da área-fonte e decrescem progressivamente em direção às bordas, foi assumida, arbitrariamente, uma distribuição de saturação de 53\% no centro da área-fonte, decrescendo até um valor de $5 \%$ nas bordas (Figura 3). Estes valores de saturação correspondem à média dentro do volume elementar representativo utilizado pelo EXHAURIS para o cálculo de transferência de massa.

Segundo Teramoto, Chang e Caetano-Chang (2017), a areia média a grossa argilosa representa a principal litologia na porção superior da zona saturada, razão pela qual optou-se por simular um modelo homogêneo, com valores de condutividade hidráulica e porosidade efetiva associadas a esta litologia. Em concordância com Teramoto et al. (2017), utilizou-se condutividade hidráulica de $6,0 \times 10^{-5} \mathrm{~m} / \mathrm{s}$. Tendo em vista que a presença do LNAPL no meio poroso promove a redução da porosidade e permeabilidade em relação à água na áreafonte, esses valores têm de ser corrigidos para reproduzir tal redução. Baseando-se na saturação de LNAPL admitida (Figura 3) e nos parâmetros da curva de retenção apresentados por Pede (2009), foi calculada a permeabilidade relativa $\left(k_{r w}\right)$, de acordo com o modelo de Mualem (1976) (Eq. 7).

$$
k_{r w}=S_{e w}^{n}\left[1-\left(1-S_{e w}^{\frac{1}{m}}\right)^{m}\right]^{2}
$$

Onde $S_{e w}=$ saturação efetiva da água, $n$ = tortuosidade do meio poroso e $m$ = parâmetro do modelo de van Genuchten (1981).

Com base nos valores de $k_{r w}$ obtidos, os valores de condutividade hidráulica no modelo foram reduzidos dentro da áreafonte para reproduzir o efeito da presença de LNAPL trapeado. Tendo em vista que a litologia da areia média a grossa argilosa, possui porosidade efetiva de 0,22 (PEDE, 2009), foi imposto este valor em todo o modelo. No caso de ausência da fase não-aquosa nos poros, foi admitido um volume unitário de água nos poros $(\omega)$ igual à porosidade efetiva. No caso da porção compreendida pela área-fonte, o volume unitário de água foi corrigido a partir da subtração do volume ocupado pelo LNAPL, de acordo com a Eq. 8.

$\omega=n_{e}\left(1-S_{n w}\right)$

O gradiente hidráulico observado de 0,0063, calculado a partir dos mapas potenciométricos de Teramoto (2015), foi imposto como condição de contorno nos limites oeste e leste do modelo, com direção de fluxo de oeste para leste.

\section{Simulação da transferência de massa}

A simulação do movimento advectivo-dispersivo de compostos monoaromáticos no modelo foi mediado pelo modelo numérico de transporte MT3DMS. A área-fonte foi assumida como 
uma condição de contorno de concentração especificada ( $\mathrm{Fi}$ gura 3). As variações de concentração dentro da área-fonte ao longo do tempo foram reproduzidas a partir do aplicativo EXHAURIS, que reproduz a sobreposição de dois efeitos, uma tendência de longo prazo, controlada pela saturação, e uma segunda, controlada pelas variações sazonais do nível d'água.

Teramoto \& Chang (2017) demonstraram que as variações das concentrações ao longo do tempo são decorrentes da sobreposição de variações sazonais, controlado pela flutuação do nível d'água, sobre uma tendência de queda de longo prazo, governada pela saturação do LNAPL no meio poroso. Tendo em vista o propósito de se avaliar apenas tendências de anos a décadas, o efeito da flutuação sazonal do NA foi suprimido a partir da imposição de uma carga constante e fixada ao longo de todo o período simulado (20 anos). Uma vez que a flutuação do nível d'água não foi representada, assumese, de maneira simplista, que não existe os recorrentes ciclos de drenagem e embebição induzidas pela flutuação do nível d'água no meio poroso contendo LNAPL. Embora esta premissa não seja verdadeira, supõe-se que para casos de LNAPL fortemente trapeados, episódios de drenagem e embebição sejam apenas episódicos.

A fração molar dos compostos BTEX foi obtida a partir da concentração desses compostos no querosene de aviação fresco, conforme apresentado por Teramoto (2015). A partir da fração molar desses compostos, foram calculadas suas solubilidades efetivas no LNAPL. A concentração dos compostos BTEX no querosene de aviação, bem como os valores de solubilidade efetiva são apresentados na Tabela 1.

Tabela 1 - Concentração de compostos BTEX no querosene de aviação e solubilidade efetiva dos compostos BTEX calculadas pela Lei de Raoult

\begin{tabular}{ccc}
\hline Composto & $\begin{array}{c}\text { Concentração no quero- } \\
\text { sene de aviação }(\mathrm{mg} / \mathrm{L})\end{array}$ & $\begin{array}{c}\text { Solubilidade efe- } \\
\text { tiva na água } \\
(\mathrm{mg} / \mathrm{L})\end{array}$ \\
\hline Benzeno & 28 & 0,1316 \\
Tolueno & 423 & 0,5066 \\
Etilbenzeno & 1199 & 0,3540 \\
m,p-xilenos & 2921 & 1,7738 \\
o-xileno & 2546 & 2,7660 \\
\hline \hline
\end{tabular}

As Tabelas 2 e 3 apresentam os valores dos parâmetros empregados no aplicativo EXHAURIS para simular as variações das concentrações de benzeno e etilbenzeno, respectivamente. Grant \& Gerhard (2007) e Potter et al. (2010), por intermédio de experimentos laboratoriais, verificaram que a área interfacial NAPL-água inicialmente cresce à medida que a saturação do NAPL é incrementada, atingindo um patamar. A partir deste patamar, a área interfacial cai progressivamente à medida que saturação do NAPL aumenta. A área-interfacial representa um parâmetro difícil de se obter em campo e, por esta razão, nas simulações realizadas, foi imposto esta relação de área-interfacial e saturação do LNAPL adotando-se como critério os valores experimentais de Potter et al. (2010).

Tabela 2 - Parametrização das simulações pelo EXHAURIS para a depleção de benzeno

\begin{tabular}{|c|c|c|c|c|c|}
\hline \multirow{2}{*}{ Parâmetro } & \multicolumn{5}{|c|}{ Saturação } \\
\hline & $5 \%$ & $12 \%$ & $22 \%$ & $38 \%$ & $53 \%$ \\
\hline $\begin{array}{l}\text { Massa do composto } \\
\text { (moles) }\end{array}$ & 0,0039 & 0,0095 & 0,0173 & 0,030 & 0,042 \\
\hline Massa de LNAPL (moles) & 53,33 & 128 & 234,67 & 405,33 & 565 \\
\hline Área Interfacial $\left(\mathrm{m}^{2}\right)$ & 50,26 & 29 & 22 & 9,68 & 8 \\
\hline$\gamma_{i, o}$ & 1 & 1 & 1 & 1 & 1 \\
\hline$V w\left(m^{3}\right)$ & 0,418 & 0,3872 & 0,3432 & 0,2728 & 0,21 \\
\hline Ki (m/dia) & $4,0 \times 10^{-4}$ & $4,0 \times 10^{-4}$ & $4,0 \times 10^{-4}$ & $4,0 \times 10^{-4}$ & $4,0 \times 10^{-4}$ \\
\hline $\mathrm{Q}\left(\mathrm{m}^{3} / \mathrm{dia}\right)$ & 0,061 & 0,057 & 0,059 & 0,051 & 0,049 \\
\hline$I\left(\operatorname{dias}^{-1}\right)$ & 0,014 & 0,014 & 0,014 & 0,014 & 0,014 \\
\hline
\end{tabular}

Tabela 3 - Parametrização das simulações pelo EXHAURIS para a depleção de etilbenzeno

\begin{tabular}{cccccc}
\hline Parâmetro & $5 \%$ & $\mathbf{1 2 \%}$ & $\mathbf{2 2 \%}$ & $\mathbf{3 8 \%}$ & $\mathbf{5 3 \%}$ \\
\hline Massa do composto (moles) & 0,124 & 0,298 & 0,547 & 0,944 & 1,316 \\
Massa de LNAPL (moles) & 53,33 & 128 & 234,67 & 405,33 & 565 \\
Área Interfacial $\left(\mathrm{m}^{2}\right)$ & 50,26 & 29 & 22 & 9,68 & 8 \\
$\gamma_{i, 0}$ & 1 & 1 & 1 & 1 & 1 \\
$V w\left(\mathrm{~m}^{3}\right)$ & 0,418 & 0,3872 & 0,3432 & 0,2728 & 0,2068 \\
$\mathrm{Ki}(\mathrm{m} / \mathrm{dia})$ & $4,0 \times 10^{-4}$ & $4,0 \times 10^{-4}$ & $4,0 \times 10^{-4}$ & $4,0 \times 10^{-4}$ & $4,0 \times 10^{-4}$ \\
$\mathrm{Q}\left(\mathrm{m}^{3} / \mathrm{dia}\right)$ & 0,0613 & 0,057 & 0,059 & 0,051 & 0,049 \\
I (dias -1$)$ & 0,014 & 0,014 & 0,014 & 0,014 & 0,014 \\
\hline
\end{tabular}


Para o cálculo da transferência de massa, o EXHAURIS requer o cálculo da solubilidade efetiva, em concordância com a Lei de Raoult. Para o cálculo da solubilidade efetiva foi empregado $\gamma_{i, o}$ igual a 1, uma vez que vários trabalhos, incluindo Cline et al., (1991), Luthy et al., (1993), Eberhardt \& Grathwohl (2002), e Chrysikopoulos \& Lee (1998), afirmam que esse parâmetro é igual ou próximo de 1 para hidrocarbonetos aromáticos.

\section{Modelo de transporte de solutos}

Dentro da proposta metodológica sumariada na Figura 2, foi empregado o aplicativo MT3DMS (ZHENG \& WANG, 1999) para simular o transporte, na área-fonte, de benzeno e etilbenzeno solubilizado em água. A Tabela 4 apresenta os parâmetros empregados nas simulações de transporte empregando o MT3DMS, baseando-se nos valores apresentados por Teramoto (2015).

Para fins de simplificação, foi empregado um fator de retardamento igual a 1. Embora Teramoto (2015) tenha obtido uma taxa de biodegradação com coeficiente de decaimento de primeira ordem de 0,0866 dias $^{-1}$ a partir de 7 anos de simulação de fluxo e transporte em regime transiente, no presente trabalho foi empregado o valor de 0,01386, para simulação de um cenário mais conservador.

Tabela 4 - Parametrização dos modelos de transporte

\begin{tabular}{cc}
\hline Parâmetro & Valor \\
\hline Dispersividade longitudinal $(\mathrm{m})$ & 2 \\
Dispersividade transversal $(\mathrm{m})$ & 0,01 \\
Dispersividade vertical $(\mathrm{m})$ & 0,001 \\
Retardamento & 1 \\
Coeficiente de decaimento primeira or- & 0,01386 \\
\hline
\end{tabular}

\section{RESULTADOS}

\subsection{Comportamento do benzeno e etilbenzeno para dife- rentes saturações do LNAPL}

As Figuras 5a e 5b apresentam, respectivamente, as variações da concentração de benzeno e etilbenzeno dentro da área-fonte, ao longo de 20 anos, para os diferentes valores de saturação por LNAPL considerados (Figura 3).

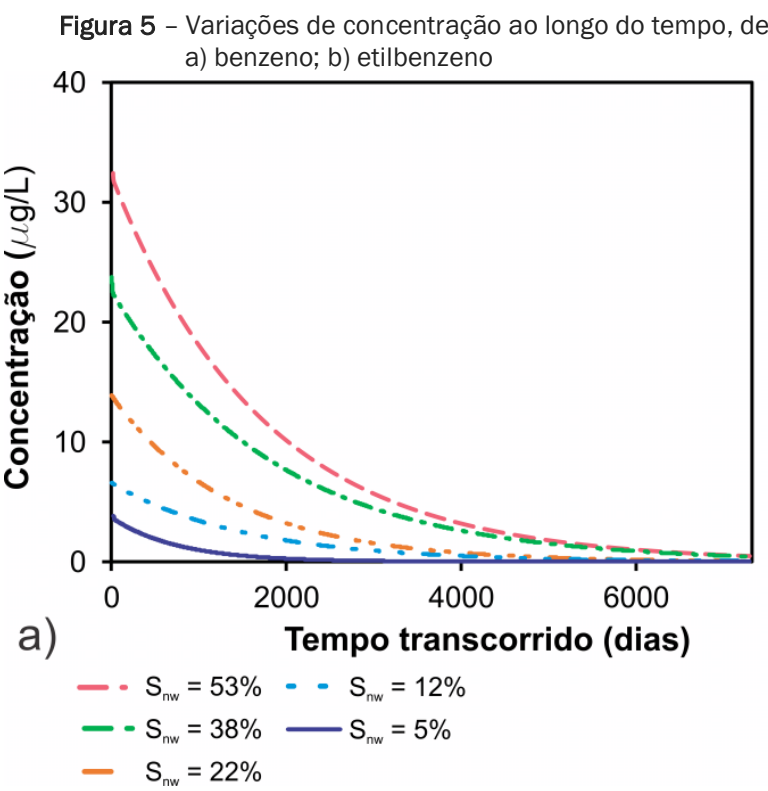

Nota-se, na Figura 5, que o tempo necessário para a depleção do LNAPL é menor em áreas com saturações baixas de LNAPL e é mais prolongado em áreas com elevadas saturações de LNAPL, em concordância com o exposto por Teramoto \& Chang (2017). Além da saturação por LNAPL, os referidos autores verificaram que o parâmetro que controla a velocidade

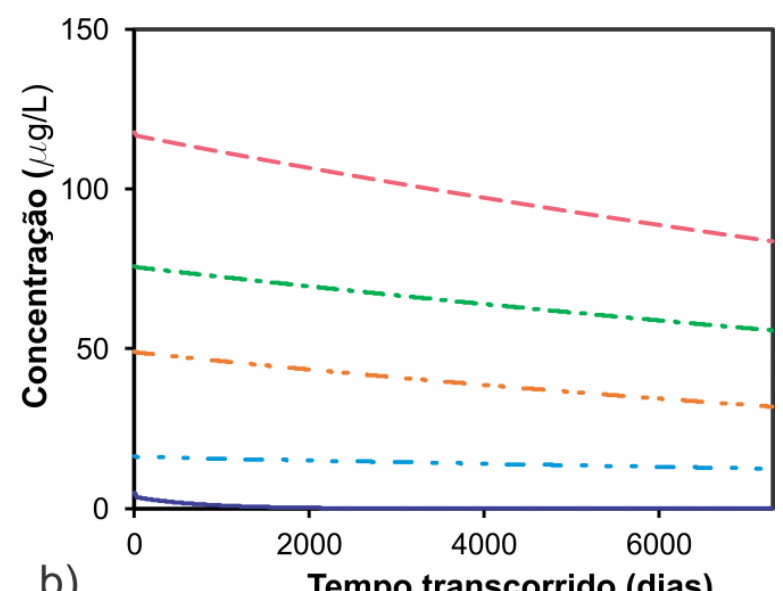

b)

Tempo transcorrido (dias)

de depleção da área-fonte é o fluxo. Para ilustrar o impacto das taxas de fluxo de água subterrânea na depleção do LNAPL, foram realizadas simulações com diversos valores de Q. (Figura 6). Os resultados indicam que o intervalo de tempo necessário para a depleção do LNAPL é mais reduzido em aquíferos com maiores velocidades de fluxo. 
Figura 6 - Curvas de queda da concentração do benzeno para três valores distintos de fluxo: a) Queda de concentrações no LNAPL;
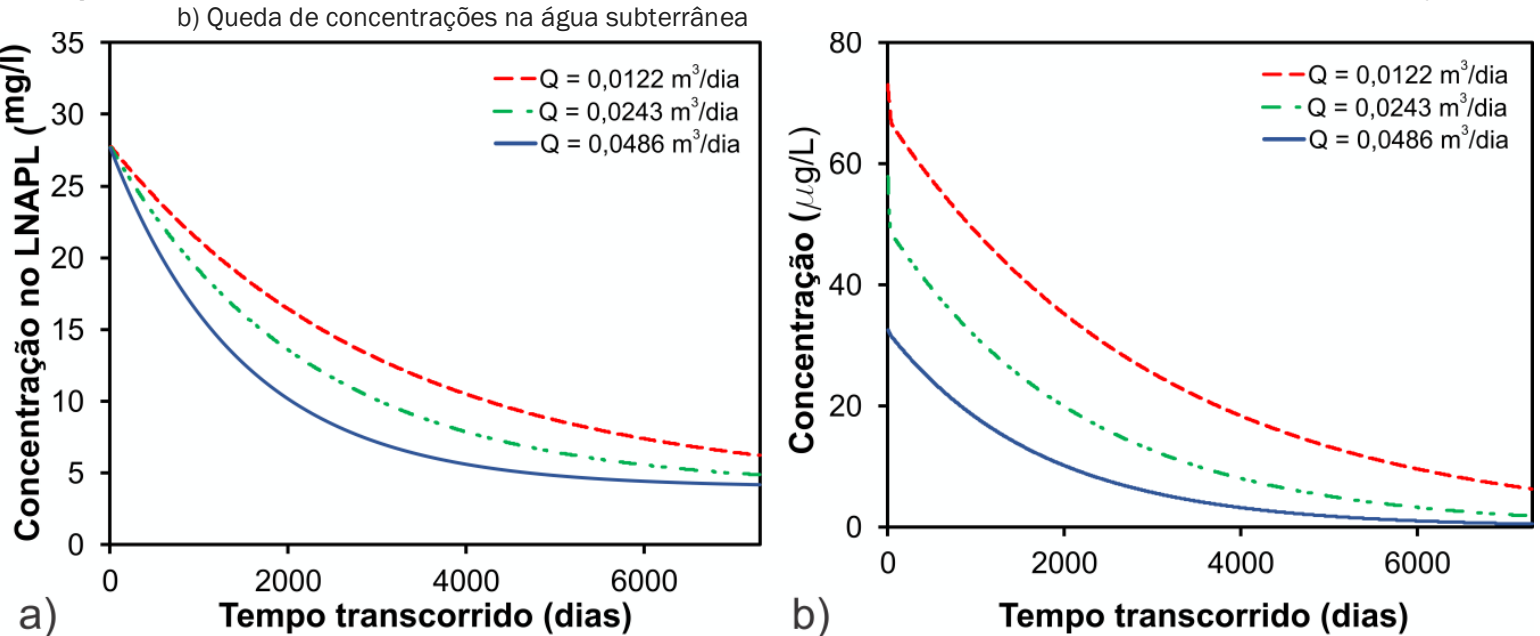

\subsection{Simulações de transporte de benzeno e etilbenzeno}

As simulações realizadas em um volume elementar representativo foram atribuídas a todas as células do modelo com valores similares de saturação por LNAPL, conforme apresentados na Figura 3. Os valores de concentração de benzeno e etilbenzeno em diferentes períodos, obtidos nas simulações rea-

lizadas no EXHAURIS, foram inseridas no MT3DMS com condições de contorno de concentração especificada; a partir deste modelo foram realizadas simulações de transporte para um período de 20 anos, empregando a parametrização apresentada na Tabela 4. As Figuras 7 e 8 apresentam, respectivamente, a evolução das plumas dissolvidas de benzeno e etilbenzeno em uma área-fonte depletiva.
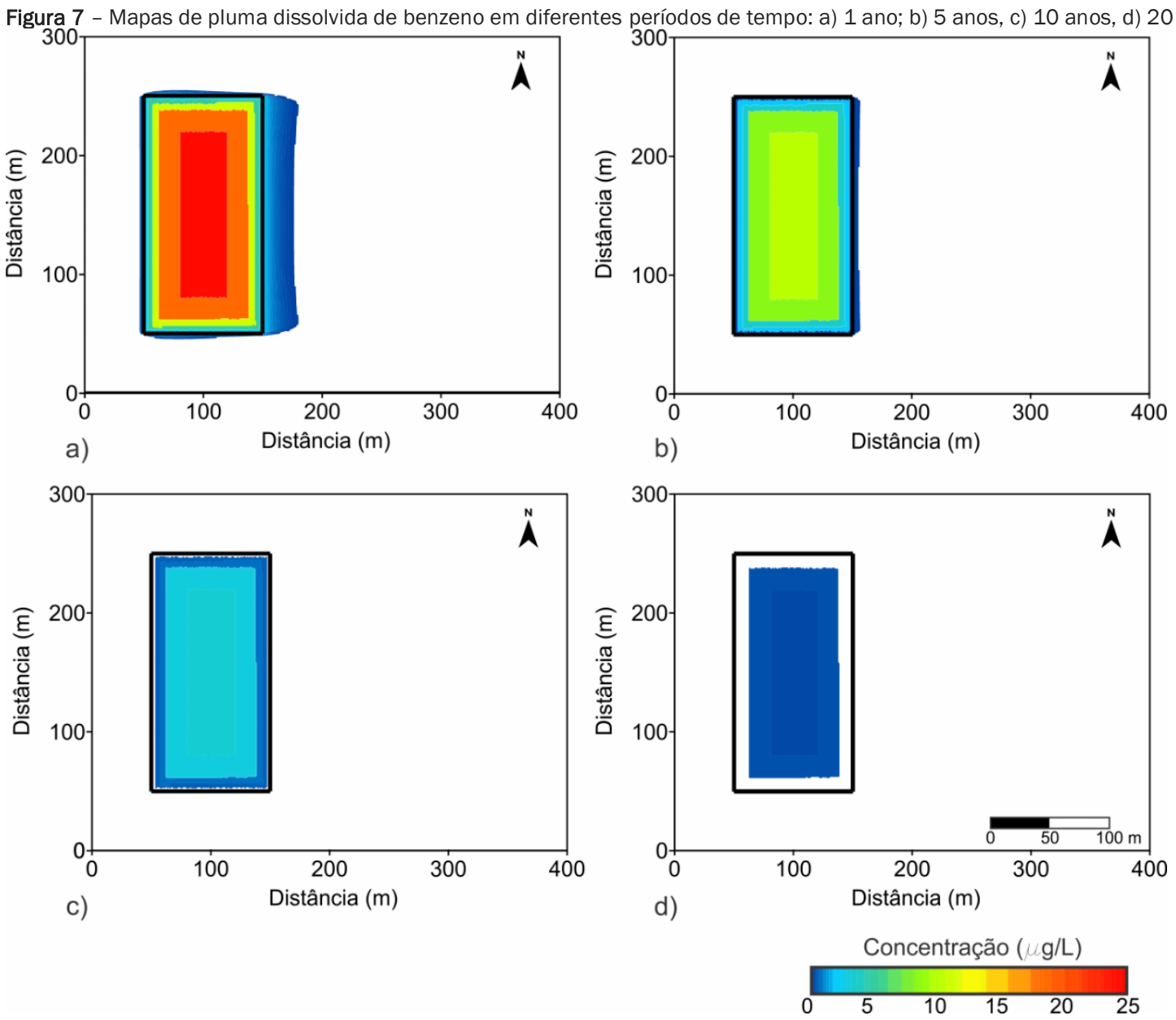

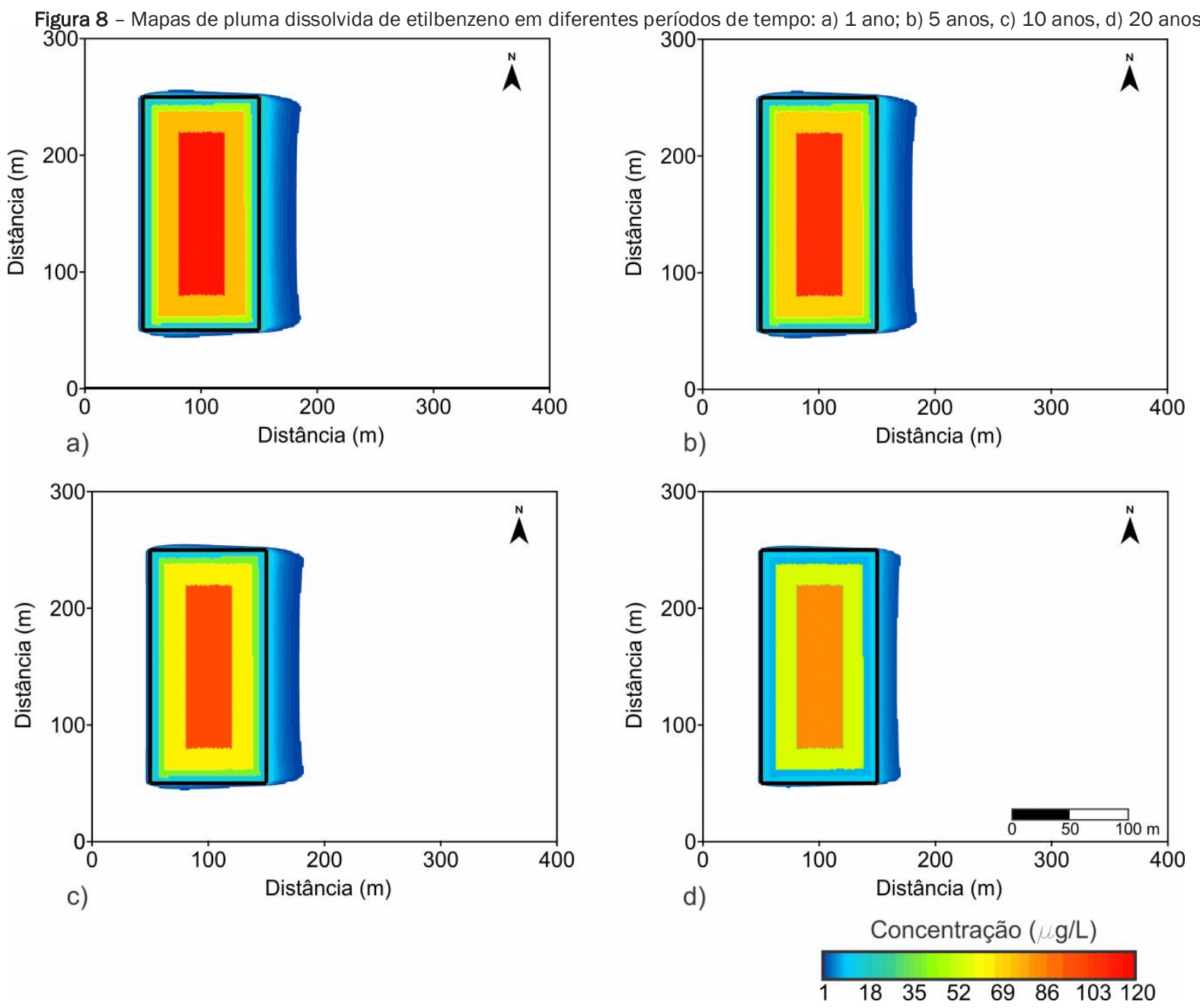

Nas simulações geradas (Figuras 7 e 8), é possivel notar que as plumas atingem suas dimensões máximas 1 ano após o início da simulação, a partir do qual se observa uma contínua tendência de redução das plumas. A pluma de benzeno desaparece completamente dentro do período de 10 anos, enquanto a pluma de etilbenzeno persiste após 20 anos. Este fato indica que as plumas dissolvidas de etilbenzeno, associadas a uma área-fonte representada por querosene de aviação, possuirão longevidade em escala de tempo de décadas. A depleção mais efetiva do benzeno está associada à sua fração molar reduzida dentro do querosene de aviação (7,36 x105), associada à sua elevada solubilidade em água (1786 $\mathrm{mg} / \mathrm{L})$. Em razão dessas características, as concentrações de benzeno apresentam uma tendência de queda exponencial ao Iongo do tempo, descrevendo um decaimento de primeira ordem, comportamento típico de um fenômeno fickiano. Na con-

dição oposta, dada a fração molar mais expressiva $(2,32$ × 10 3) e menor solubilidade (187 mg/L), o etilbenzeno será depletado menos eficientemente. Em razão de sua baixa taxa de transferência de massa, a queda nas concentrações do etilbenzeno aparenta seguir uma tendência linear dentro da escala de tempo simulado, embora se trate de uma queda exponencial.

Nota-se que os resultados deste trabalho estão em concordância com as análises da concentração de compostos BTEX no LNAPL apresentados por Teramoto (2015) (Tabela 5). Esse autor verificou que em 9 amostras de LNAPL o benzeno estava depletado em 6, enquanto o etilbenzeno ainda apresentava concentrações significativas em todas as amostras, quando comparadas com a amostra de querosene de aviação fresco. 
Tabela 5 - Concentração de compostos BTEX nas amostras de LNAPL e do querosene de aviação fresco, apresentados por Teramoto (2015)

\begin{tabular}{ccccc}
\hline ID & $\begin{array}{c}\text { Benzeno } \\
(\mathrm{mg} / \mathrm{L})\end{array}$ & $\begin{array}{c}\text { Tolueno } \\
(\mathrm{mg} / \mathrm{L})\end{array}$ & $\begin{array}{c}\text { Etilbenzeno } \\
(\mathrm{mg} / \mathrm{L})\end{array}$ & $\begin{array}{c}\text { Xilenos totais } \\
(\mathrm{mg} / \mathrm{L})\end{array}$ \\
\hline RE-PM-53 & $<1$ & 13 & 832 & 3848 \\
RE-PM-63 & $<1$ & 23 & 642 & 3942 \\
RE-PM-65 & $<1$ & 59 & 603 & 4707 \\
RE-PM-75 & $<1$ & 12 & 370 & 4315 \\
RE-PM-86 & 22 & 112 & 1271 & 4826 \\
RE-PM-95 & $<1$ & 56 & 1154 & 4783 \\
RE-PM-98 & $<1$ & $<1$ & 188 & 1099 \\
RE-PM-115A & 16 & 132 & 1096 & 4857 \\
RE-PM-99A & 3 & $<1$ & 1036 & 3174 \\
QAV fresco & 28 & 423 & 1199 & 5467 \\
\hline
\end{tabular}

\section{DISCUSSÕES}

A implantação da rede de monitoramento RIMAS (Rede Integrada de Monitoramento de Águas Subterrâneas) pela CPRM (Serviço Geológico do Brasil), ofereceu a oportunidade de avaliar o comportamento sazonal do nível d'água em diferentes regiões do território brasileiro, contemplando condições geológicas, climáticas e hidrológicas distintas. A simples consulta a alguns poços desta rede de monitoramento permite constatar serem comuns variações de $2 \mathrm{~m}$ a $3 \mathrm{~m}$, quando os dados de 4 ou 5 anos consecutivos estão disponíveis. Tal magnitude é similar à apresentada por Teramoto \& Chang (2018) em Paulínia (SP). Face a esta variação, o LNAPL encontra condições favoráveis para seu trapeamento, uma vez que quando o NA cai, o LNAPL migra para porções mais baixas e se mantém aprisionado por capilaridade quando o NA sobe. Na condição de trapeamento, o LNAPL não possui mobilidade e não migra para os poços de monitoramento e, consequentemente, não há registro de fase livre no interior desses poços. A simples ausência de fase livre suscita o equivocado diagnóstico que as elevadas concentrações, mensuradas em amostras de água subterrâneas, estão relacionadas à migração de plumas dissolvidas com elevadas concentrações.

Na investigação de áreas contaminadas, a área-fonte nunca foi devidamente caracterizada em razão de dois fatores preponderantes. 0 primeiro decorre de erros conceituais, na qual se assumia que a simples ausência de fase livre nos poços de monitoramento indica a inexistência de LNAPL. O segundo é representado pela difundida prática de realizar análises de solos apenas na porção não-saturada e na franja capilar, quando apenas análises na zona saturada podem fornecer indicativos de LNAPL trapeado. 0 advento de ferramentas de alta resolução alterou esta percepção, como pontuado por Suthersan et al. (2015), Gatsios et al. (2018) e Isler et al. (2018).

Em razão da ampla variação do nível d'água ao longo do tempo é esperado que, em casos de contaminações antigas, o LNAPL esteja trapeado na maior parte do tempo e apenas em condições episódicas, quando o NA cai significantemente, este seja liberado e ganhe mobilidade. A maior parte dos modelos conceituais de contaminação não contemplam a condição de trapeamento, fazendo com que os diagnósticos e ações para a remediação sejam normalmente falhos. Na condição de trapeamento, a transferência de massa para a água é o principal mecanismo para a depleção do LNAPL em relação aos compostos aromáticos. Nesses casos, os modelos de contaminação devem contemplar o efeito depletivo do LNAPL para previsões de cenários mais realistas.

Cabe ressaltar, entretanto, que em casos de vazamentos recentes, o LNAPL pode ainda não ter sido redistribuído para porções mais profundas pela flutuação do nível d'água e que sua maior esteja acumulada na porção superior da zona saturada. Nesses casos, parcela significativa do LNAPL está distribuída na zona não-saturada e a volatização é o mecanismo mais efetivo na depleção do LNAPL.

As simulações apresentadas no presente trabalho apontam que o benzeno é rapidamente depletado e o etilbenzeno persiste por períodos prolongados. A depleção mais efetiva do benzeno aqui observada é similar ao apresentado no experimento numérico conduzido por Thornton et al. (2013), que demonstrou que o benzeno é o primeiro composto a ser depletado no LNAPL, e o etilbenzeno, o composto com maior longevidade. Similarmente, a depleção mais efetiva do benzeno é compatível com os resultados de Teramoto (2015), que demonstrou que em 6 das 9 amostras de LNAPL (Tabela 5) em uma área contaminada por querosene de aviação, a concentração do benzeno estava abaixo do limite de detecção, enquanto o etilbenzeno ocorria em elevadas concentrações.

A taxa de queda nas concentrações na fase aquosa é controlada por diversos fatores, tais como velocidade de fluxo, saturação do LNAPL, área interfacial LNAPL/água e taxa de biodegradação. Análises de sensibilidade indicam que o fator mais importante é a taxa de fluxo (Q) dentro do volume elementar representativo. Como ilustrado na Figura $6 \mathrm{a}$, incrementos nas taxas de fluxo promovem fortes variações na velocidade de depleção do LNAPL, fazendo com que a concentração na fase aquosa igualmente caia (Figura 8b). Por esta razão é esperado que a retração da pluma dissolvida ao longo do tempo, em razão da depleção da área-fonte, seja mais rápida em aquíferos com elevadas velocidades de fluxo e, consequentemente, menos intensas em aquíferos com taxas de fluxo reduzidas. 
Outro fator igualmente importante para a velocidade de depleção do LNAPL é sua saturação no meio poroso, como se pode observar na Figura 5. Ebehardt et al. (2002) e Teramoto \& Chang (2017) demonstraram que elevadas saturações de LNAPL podem sustentar concentrações elevadas em escala de tempo de décadas, enquanto baixas saturações de LNAPL apresentam completa depleção em alguns anos.

A dificuldade de simular a transferência de massa interfases está relacionada à aquisição dos parâmetros requeridos, uma vez que tais parâmetros não são obtidos em investigações ambientais, particularmente a composição e a saturação do LNAPL. Os modelos analíticos de Jeong \& Chaberneau (2014) ou de Lenhard et al. (2018) permitem estimar o perfil de saturação a partir das espessuras de fase livre. Contudo, tais modelos exigem um longo período de monitoramento para se determinar a máxima espessura de fase livre registrada nos poços de monitoramento, bem como parâmetros obtidos a partir de ensaios de curva de retenção em amostras indeformadas, que são difíceis de serem obtidas em profundidade. A quantificação da saturação do LNAPL pode ser obtida a partir da extração por destilação Dean Stark, descrita em API (1998), em que o volume de hidrocarbonetos na amostra de solo é determinado a partir de sua destilação. Uma alternativa usual é a estimativa a partir das análises de TPH (Totais de Hidrocarbonetos de Petróleo) da Equação proposta por Parker et al., (1994) (Eq, 9), que leva em consideração a densidade e a porosidade do aquífero.

$S_{n w}=\frac{\rho_{b} \cdot T P H}{\rho_{n} \cdot \emptyset \cdot 10^{6}}$

Onde $S_{n w}=$ saturação do NAPL, $\rho_{b}=$ densidade aparente seca do solo $\left(\mathrm{g} / \mathrm{cm}^{3}\right)$, TPH $=$ quantidade de totais de petróleo (mg/kg), $\rho_{n}=$ densidade do NAPL $\left(\mathrm{g} / \mathrm{cm}^{3}\right)$ e $\emptyset=$ porosidade.

Uma vez transportada por movimento advectivo, as plumas dissolvidas alcançarão um tamanho máximo que é determinado pelas taxas de biodegradação atuantes. Desse modo, as dimensões pouco expressivas das plumas dissolvidas geradas nas simulações (Figuras 7 e 8) refletem os elevados coeficientes de primeira ordem empregados no modelo. Teramoto (2015) verificou que a partir de 8 anos de monitoramento as plumas de fase dissolvida de compostos BTEX não apresentavam tendência de expansão, refletindo elevadas taxas de biodegradação.

Os resultados aqui apresentados demonstram a necessidade de investigar a composição do LNAPL e monitorar as concentrações na fase aquosa para previsões mais realistas da longevidade da área-fonte. Dentro do contexto apresentado pelo presente trabalho é necessária especial atenção à caracterização da condição de intemperismo do LNAPL no gerenciamento de áreas contaminadas.

\section{CONCLUSÕES}

Baseando-se no conceito da depleção do LNAPL em fase residual da zona saturada, foi aqui proposta uma metodologia de simulação que contempla a contínua queda da fração molar dos compostos BTEX no LNAPL, com vistas a previsões mais realísticas. Os resultados indicam a viabilidade da metodologia proposta, que constitui uma alternativa estratégica para previsão do movimento de plumas dissolvidas a partir de modelos numéricos de transporte. Os resultados deste trabalho evidenciam que o benzeno é rapidamente depletado, em razão de sua elevada solubilidade, fazendo com que as plumas dissolvidas deste composto possuam longevidade reduzida. Por outro lado, o etilbenzeno é depletado de maneira menos eficiente, de forma que a pluma dissolvida deste composto mostra longevidade com escala de tempo de décadas.

\section{REFERÊNCIAS}

API. Recommended Practices for Core Analysis, 2nd Edition, Document Number: API RP 40, American Petroleum Institute 200 p, 1998.

BANERJEE, Sujit, Solubility of organic mixtures in water. Environmental Science \& Technology, v. 18, n. 8, p. 587-591, 1984.

CHRYSIKOPOULOS, Constantinos V.; LEE, Kenneth Y. Contaminant transport resulting from multicomponent nonaqueous phase liquid pool dissolution in three-dimensional subsurface formations. Journal of Contaminant Hydrology, v. 31, n, 1-2, p. 1-21, 1998.

CLINE, Patricia V.; DELFINO, Joseph J.; RAO, P. Suresh C. Partitioning of aromatic constituents into water from gasoline and other complex solvent mixtures. Environmental Science \& Technology, v. 25, n. 5, p. 914-920, 1991.

CONNOR, John A, et al, Review of quantitative surveys of the length and stability of MTBE, TBA, and benzene plumes in groundwater at UST sites. Groundwater, v, 53, n, 2, p, 195206, 2015.

EBERHARDT, Christina; GRATHWOHL, Peter, Time scales of organic contaminant dissolution from complex source zones: coal tar pools vs. blobs. Journal of Contaminant Hydrology, v. 59 , n, 1-2, p, 45-66, 2002.

FARR, Ann M.; HOUGHTALEN, R. J.; MCWHORTER, D. B. Volume estimation of light nonaqueous phase liquids in porous media. Groundwater, v. 28, n. 1, p. 48-56, 1990.

FLORES, Giancarlo et al. Migration of different LNAPLs in subsurface under groundwater fluctuating conditions by the simplified image analysis method. Journal of Geo-Engineering Sciences, v. 3, n. 1, p. 15-30, 2016.

GARG, Sanjay et al. Overview of natural source zone depletion: Processes, controlling factors, and composition change. Groundwater Monitoring \& Remediation, v. 37, n. 3, p. 62-81, 2017.

GRANT, G. P.; GERHARD, J. I. Simulating the dissolution of a complex dense nonaqueous phase liquid source zone: 1. Model to predict interfacial area. Water Resources Research, v. 43, n. 12, 2007.

GATSIOS, Evangelos et al. LNAPL transmissivity as a remediation metric in complex sites under water table fluctuations. Journal of environmental management, v. 215, p. 4048, 2018.

HARBAUGH, Arlen W. MODFLOW-2005, the US Geological Survey modular ground-water model: the ground-water flow process. Reston, VA: US Department of the Interior, US Geological 
Survey, 2005.

ISLER, Elias et al, Trapeamento de LNAPL observado por meio da técnica de fluorescência induzida por laser (LIF). Águas Subterrâneas, v. 32, n. 3, p. 315-324, 2018.

JEONG, Jaehak; CHARBENEAU, Randall J. An analytical model for predicting LNAPL distribution and recovery from multi-layered soils. Journal of contaminant hydrology, v. 156, p. 52-61, 2014.

KEMBLOWSKI, M. W.; CHIANG, C. Y. Hydrocarbon thickness fluctuations in monitoring wells. Groundwater, v, 28, n, 2, p, 244-252, 1990.

KULKARNI, Poonam R, et al. Evaluation of source-zone attenuation at LUFT sites with mobile LNAPL. Soil and Sediment Contamination: An International Journal, v. 24, n. 8, p. $917-$ 929, 2015.

LENHARD, Robert J. et al. Evaluating an analytical model to predict subsurface LNAPL distributions and transmissivity from current and historic fluid levels in groundwater wells: comparing results to numerical simulations. Groundwater Monitoring \& Remediation, v. 38, n. 1, p. 75-84, 2018.

LIU, Lihua et al, Contaminant mass transfer from NAPLs to water studied in a continuously stirred flow-through reactor. Journal of Environmental Engineering, v. 138, n. 8, p. 826-832, 2012.

LUTHY, Richard G, et al, Interfacial films in coal tar nonaqueous-phase liquid-water systems. Environmental science \& technology, v. 27, n. 13, p. 2914-2918, 1993.

MACKAY, Douglas et al, Comparing Natural Source Zone Depletion Pathways at a Fuel Release Site. Groundwater Monitoring \& Remediation, v. 38, n. 2, p. 24-39, 2018.

MACKAY, Donald et al, Dissolution of non-aqueous phase liquids in groundwater. Journal of Contaminant Hydrology, v. 8 , n, 1, p. 23-42, 1991.

MILLER, Cass T.; POIRIER-MCNEIL, Michele M.; MAYER, Alex S. Dissolution of trapped nonaqueous phase liquids: Mass transfer characteristics. Water Resources Research, v. 26, n. 11, p. 2783-2796, 1990.

MARINELLI, Fred; DURNFORD, Deanna S. LNAPL thickness in monitoring wells considering hysteresis and entrapment. Groundwater, v. 34, n. 3, p. 405-414, 1996.

MUALEM, Yechezkel. A new model for predicting the hydraulic conductivity of unsaturated porous media. Water resources research, v.,12, n. 3, p. 513-522, 1976.

PARKER, Jack C,; WADDILL, Dan W,; JOHNSON, Jeffrey A, UST corrective action technologies: Engineering design of free product recovery systems, Environmental Systems and Technologies, 1994.
PEDE, Marco Aurelio Zequim. Flutuação do lençol freático e sua implicação na recuperação de hidrocarbonetos: um estudo de caso. Tese (Doutorado)- Instituto de Geociências e Ciências Exatas, Universidade Estadual Paulista, Rio Claro, 141 f, 2009.

PORTER, Mark L. et al. Measurement and prediction of the relationship between capillary pressure, saturation, and interfacial area in a NAPL-water-glass bead system. Water Resources Research, v. 46, n. 8, 2010.

SUTHERSAN, Suthan; KOONS, Brad; SCHNOBRICH, Matthew, Contemporary management of sites with petroleum LNAPL presence. Groundwater Monitoring \& Remediation, v. 35, n. 1, p. 23-29, 2015.

TERAMOTO, E. H. Estudo da efetividade da atenuação natural de compostos BTEX em área contaminada por querosene de aviação. Tese (Doutorado) - Instituto de Geociências e Ciências Exatas, Universidade Estadual Paulista, Rio Claro, 205 f, 2015.

TERAMOTO, Elias Hideo; CHANG, Hung Kiang. Field data and numerical simulation of BTEX concentration trends under water table fluctuations: Example of a jet fuel-contaminated site in Brazil. Journal of Contaminant Hydrology, v. 198, p. 37-47, 2017.

TERAMOTO, Elias Hideo; CHANG, Hung Kiang; CAETANOCHANG, Maria Rita. Transporte de solutos em diferentes cenários geológicos gerados por modelos estocásticos de cadeias de Markov. Águas Subterrâneas, v. 31, n. 4, p. 316-326, 2017.

TERAMOTO, Elias Hideo; CHANG, Hung Kiang. Métodos WTF e simulação numérica de fluxo para estimativa de recargaexemplo Aquífero Rio Claro em Paulínia/SP. Águas Subterrâneas, v. 32, n. 2, p. 173-180, 2018.

TERAMOTO, Elias Hideo; CHANG, Hung Kiang. Geochemical conceptual model of BTEX biodegradation in an iron-rich aquifer. Applied Geochemistry, v. 100, p. 293-304, 2019.

THORNTON, Steven F.; TOBIN, Kevin; SMITH, Jonathan WN. Comparison of Constant and Transient-Source Zones on Simulated Contaminant Plume Evolution in Groundwater: Implications for Hydrogeological Risk Assessment. Groundwater Monitoring \& Remediation, v. 33, n. 3, p. 78-91, 2013.

ZHENG, Chunmiao; WANG, P. Patrick. MT3DMS: a modular three-dimensional multispecies transport model for simulation of advection, dispersion, and chemical reactions of contaminants in groundwater systems; documentation and user's guide. Alabama Univ University, 1999. 